OPEN ACCESS

Edited by:

Barbara Altieri,

University Hospital of Wuerzburg,

Germany

Reviewed by:

Mick Welling,

Leiden University Medical Center,

Netherlands

Pasqualino Malandrino,

University of Catania, Italy

*Correspondence:

Zhao Wang

wangzhao@mail.sysu.edu.cn

Guanghua Li

ligh26@mail.sysu.edu.cn

${ }^{t}$ These authors have contributed equally to this work and share first authorship

Specialty section:

This article was submitted to

Cancer Endocrinology,

a section of the journal

Frontiers in Endocrinology

Received: 18 June 2021 Accepted: 16 August 2021 Published: 01 September 2021

Citation:

Zhou Z, Wang Z, Zhang B, Wu Y, Li G and Wang Z (2021) Comparison of 68Ga-DOTANOC and 18F-FDG PET-CT Scans in the

Evaluation of Primary Tumors and Lymph Node Metastasis in Patients With Rectal Neuroendocrine Tumors.

Front. Endocrinol. 12:727327. doi: 10.3389/fendo.2021.727327

\section{Comparison of 68Ga-DOTANOC and 18F-FDG PET-CT Scans in the Evaluation of Primary Tumors and Lymph Node Metastasis in Patients With Rectal Neuroendocrine Tumors}

\author{
Zhihao Zhou ${ }^{1+}$, Zhixiong Wang ${ }^{1+}$, Bing Zhang ${ }^{2}$, Yanzhang $\mathrm{Wu}^{1}$, Guanghua $\mathrm{Li}^{1 *}$ \\ and Zhao Wang $^{1 *}$ \\ ${ }^{1}$ Department of Gastrointestinal Surgery, First Affiliated Hospital of Sun Yat-sen University, Guangzhou, Guangdong, China, \\ 2 Department of Nuclear Medicine, First Affiliated Hospital of Sun Yat-sen University, Guangzhou, Guangdong, China
}

Background: Lymph node metastasis of rectal neuroendocrine tumors (RNETs) predicts poor prognosis. However, the assessment of lymph node metastasis remains a challenge. It has been reported that 68Ga-DOTANOC and 18F-FDG PET-CT scans could be employed in the work-up of rectal neuroendocrine tumors (RNETs). This study aimed to assess both tracers' ability to identify primary tumors and lymph node $(\mathrm{LN})$ metastasis in RNETs.

Methods: A total of 537 patients with RNETs were enrolled from January 2014 to January 2021. Both 68Ga-DOTANOC and 18F-FDG PET-CT scans were used to evaluate primary tumors and LN group metastasis. PET images were evaluated through visual and semiquantitative assessment. Receiver Operating Characteristics (ROC) curve analysis was used to investigate the performance of SUVmax of 68Ga-DOTANOC and 18F-FDG PET in predicting LN group metastasis.

Results: Fifty-two patients with preoperative 68Ga-DOTANOC with 18F-FDG PET-CT scans underwent endoscopic biopsy or dissection of the primary tumor, while 11 patients underwent rectal surgery together with regional LN dissection. For primary tumors, 68GaDOTANOC had a sensitivity of $89.58 \%$ and a positive predictive value (PPV) of $95.56 \%$ through visual assessment, while 18F-FDG PET-CT showed $77.08 \%$ sensitivity and 97.37\% PPV. For the prediction of LN group metastasis, 68Ga-DOTANOC PET-CT had $77.78 \%$ sensitivity and $91.67 \%$ specificity, while 18F-FDG PET-CT had $38.89 \%$ sensitivity and $100 \%$ specificity according to visual assessment. The area under the ROC curves (AUC) for 68Ga-DOTANOC PET/CT was 0.852 (95\%Cl:0.723-0.981) with an optimal SUVmax cut-off value of 2.25, while the AUC for 18F-FDG PET were 0.664 (95\% Cl:0.415-0.799) with an optimal SUVmax cut-off value of 1.05 . 


\section{Conclusions: This study showed that 68Ga-DOTANOC PET-CT was a promising tool for detecting LN metastasis in RNETs with high sensitivity and specificity in visual assessment and semiquantitative assessment, which was better than 18F-FDG PET-CT.}

Keywords: rectal neuroendocrine tumors, lymph node metastasis, 68Ga-DOTANOC PET, 18F-FDG PET, PET-CT

\section{INTRODUCTION}

Neuroendocrine tumors (NETs) are considered rare tumors and constitute only $0.5 \%$ of all malignant conditions (1). NETs can arise in different organs, including the gastrointestinal tract, pancreas, lungs, gallbladder, thymus, thyroid gland, testes, ovary, and skin (2). Rectal NETs (RNETs) only account for $1 \%$ to $2 \%$ of rectal tumors (3). In 2010, the World Health Organization proposed that rectal neuroendocrine tumors (NETs) are classified as malignant tumors (4), and the 5-year survival rates for RNETs were $64.1 \%$ and $88 \%$ in Europe and North America, respectively (5-7).

RNETs were mostly limited to local (8) and endoscopic dissection for most cases, which was sufficient (9). However, lymph node (LN) metastases were found in nearly $10 \%$ of cases (10). Surgery with lymphadenectomy represents the gold standard for the curative treatment of localized disease with LN metastasis (11). Although tumor size, endoscopic aspect, CT appearance, etc. could predict LN metastasis (12), how to diagnose LN metastases accurately remains uncertain.

NETs typically express somatostatin receptors (SSTRs) on their cell membranes (13). Due to the high expression of SST in most NETs, SST imaging has become the current standard for staging and preoperative assessment of NET patients $(14,15)$. Of all methods available, PET-CT with 68Ga-labeled somatostatin analogs (SSAs) (68Ga-DOTATATE, 68Ga-DOTANOC, and $68 \mathrm{Ga}-\mathrm{DOTATOC}$ ) showed the best mix of diagnostic accuracy (16). However, the predictive value of LN metastasis for SST imaging remains to be explored.

FDG is a glucose analog that is actively transported into the cell and subsequently remains in the cell. Tumor cells, due to their higher metabolic activity, usually have a higher FDG uptake than normal tissues (17). 18F-FDG PET/CT was considered the preferred radiotracer for G3 tumors, as well as for some highgrade G2 tumors (18). 18F-FDG PET-CT had a high diagnostic accuracy to identify progression in enteropancreatic NETs (19) and was a useful tool to predict the therapeutic effect in patients who underwent peptide receptor radionuclide therapy (20). However, 18F-FDG PET-CT shows high false negative findings, which could be related to the indolent tumor behavior of most NETs, such as G1 and low G2 tumors (21). The diagnostic role of 18F-FDG PET-CT is still controversial due to these conflicting results (22).

Abbreviations: NETs, neuroendocrine tumors; NENs, neuroendocrine neoplasms; RNETs, Rectal NETs; LN, lymph node; SSAs, somatostatin analogs; TNM, tumor node metastasis; SUVmax, maximum standard uptake value; IQRs, interquartile ranges; ROC, Receiver Operating Characteristics; CI, confidence interval; ESD, endoscopic submucosal dissection; PPV, positive predictive value; NPV, negative predictive value; AUC, The area under the ROC curves.
68Ga-DOTANOC PET-CT seems to be superior to 18F-FDG PET-CT in the diagnostic performance of primary and LN metastases of pancreatic NETs $(23,24)$. However, whether 68Ga-DOTANOC PET-CT can identify primary tumors and LN metastasis better than 18F-FDG PET-CT in patients with RNETs remains unclear.

In our study, we explored the diagnostic ability of $68 \mathrm{Ga}$ DOTANOC PET-CT and 18F-FDG PET-CT for RNET primary tumors and regional LN group metastasis.

\section{MATERIALS AND METHODS}

\section{Patients}

Patients who were diagnosed with RNETs from January 2014 to January 2021 were enrolled. The study was approved by the Ethics Committee of the First Affiliated Hospital of Sun Yat-sen University in China. All research was undertaken following the provisions of the Declaration of Helsinki. Patients with the following criteria were included: (1) confirmed RNETs according to 2019 World Health Organization (WHO) digestive system tumor classification criteria; (2) underwent 68Ga-DOTANOC PET-CT and 18F-FDG PET-CT within a 1month period; and (3) absence of therapeutic intervention or change in disease status between the two PET studies. The exclusion criteria were as follows: (1) other colorectal malignancies; (2) uncertain diagnosis lacking pathology; and (3) long-acting radiolabeled somatostatin analog treatment in the 4 weeks prior to the study (25). All patients provided written informed consent and complied with the ethical guidelines in the Declaration of Helsinki.

\section{Pathological Diagnosis}

The histological type of rectal NETs was defined according to the 2019 WHO classification (26), and tumor node metastasis (TNM) staging was characterized in our study according to the 2017 AJCC 8th edition (27). All NETs were graded according to the current guidelines of the 2019 WHO classification system based on mitotic counts and the Ki-67 labeling index. G1, G2, and G3 were classified according to the Ki-67 index. In brief, G1 was assigned to tumors with a mitotic rate $<2 / 10 \mathrm{HPFs}$ and/or a Ki-67 labeling index $<3 \%, \mathrm{G} 2$ to tumors with a mitotic rate 2 to $20 / \mathrm{HPFs}$ and/or a Ki-67 labeling index of $3 \%$ to $20 \%$, and G3 to tumors with a mitotic rate $>20 / \mathrm{HPFs}$ and/or a Ki-67 labeling index $>20 \%$.

\section{LN Group Classification}

According to the Japanese Classification of Colorectal, Appendiceal, and Anal Carcinoma (28), regional LNs were mainly classified into pericolic, intermediate, main LN group, 
and lateral LN groups. For some cases, the surgeon selected the enlarged LN individually during surgery according to preoperative imaging.

\section{Reference Standard}

Histopathology was taken as the reference standard.

\section{Ga-DOTANOC and 18F-FDG PET-CT Imaging}

As Qiao He reported (29), no specific preparation of the patients was required before 68Ga-DOTANOC PET-CT and 18F-FDG examination. PET-CT imaging was performed with a Gemini GXL 16 PET scanner (Philips Healthcare). One hundred eleven to $185 \mathrm{MBq}(3-5 \mathrm{mCi}) 68 \mathrm{Ga}$-DOTANOC or a dose of $5.18 \mathrm{MBq}$ $(0.14 \mathrm{mCi}) / \mathrm{kg}$ FDG was injected intravenously, and serial scanning was performed. Serial scanning from head to midthigh was performed approximately $45-60 \mathrm{~min}$ after the injection. Following low radiation dose CT acquisition with a slice thickness of $5 \mathrm{~mm}$, PET acquisition was performed for $1.5 \mathrm{~min}$ per bed position for 7-8 beds using a slice thickness of $4 \mathrm{~mm}$. CT-based attenuation correction of the emission data was employed. PET images were reconstructed by the Line of Response RAMLA algorithm.

18F-FDG and 68Ga-DOTANOC PET-CT studies were performed at least $24 \mathrm{~h}$ apart.

\section{Image Analysis}

68Ga-DOTANOC and 18F-FDG PET-CT images were evaluated both visually and semi-quantitatively by two experienced nuclear medicine physicians in consensus. For the PET-CT studies, areas with focal activity greater than the background that could not be identified as physiological activity were considered to indicate tumor tissue (defined as visual assessment). The location and radioactivity uptake (maximum standard uptake value, SUVmax) of the lesions were observed or measured (defined as semiquantitative analysis).

\section{Statistical Analysis}

Normally distributed variables were expressed as the means and standard deviations (SD), with variables not following a normal distribution presented as medians and interquartile ranges (IQRs) and categorical variables as frequencies and proportions. The Shapiro-Wilk test was used to test deviations from a normal distribution. The nonparametric analyses were performed using the Mann-Whitney $\mathrm{U}$ test. A receiver operating characteristic (ROC) curve was then constructed to determine the optimal SUVmax cutoff for predicting LN metastasis. A p value of $<0.05$ was considered statistically significant. The data analysis was performed using GraphPad Prism 8 software (GraphPad Software, Inc., La Jolla, CA, USA) and MedCalc statistical software.

\section{RESULTS}

\section{Patients Characteristics}

A total of 52 patients who underwent both 68Ga-DOTANOC and 18F-FDG PET-CT scans were included in the study. Of the 11 patients who underwent regional $\mathrm{LN}$ dissection, 1 patient underwent salvage surgery after endoscopic submucosal dissection (ESD), 5 patients with distant metastases underwent surgery when intestinal obstruction occurred, and the other 5 patients underwent radical surgery. Another 41 patients underwent endoscopic biopsy or dissection only. More details are shown in Figure $\mathbf{1}$ and Table $\mathbf{1 .}$

\section{Comparison of the Performance of $68 \mathrm{Ga}$ - DOTANOC and 18F-FDG PET-CT in Primary Tumors}

\section{Visual Assessment}

Of the 52 patients included, 48 patients were pathologically diagnosed with NETs for the primary tumor, while the remaining 4 patients were determined to be negative by pathology for the primary tumor due to preoperative endoscopic dissection.

68Ga-DOTANOC PET-CT successfully identified 43/48 primary tumors with a sensitivity of $89.58 \%$ and $95.56 \% \mathrm{PPV}$, while 18 F-FDG PET-CT identified $37 / 48$ primary tumors with a

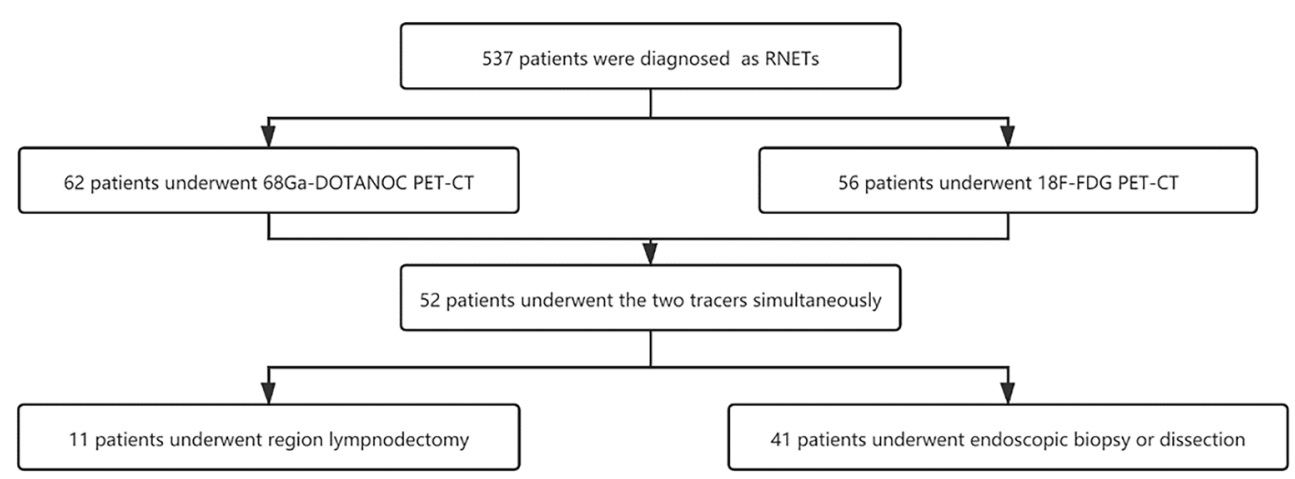

FIGURE 1 | The flowchart of study patients. 
TABLE 1 | Basic clinicopathological characteristics in patients who underwent 68Ga-DOTANOC and 18F-FDG PET-CT.

\begin{tabular}{lc}
\hline Characteristics & value \\
\hline Sex & \\
Male & 32 \\
Female & 20 \\
Age (years) & $28-75$ \\
TNM stage & \\
I-III & 11 \\
IV & 41 \\
Grade & \\
G1 & 15 \\
G2 & 31 \\
G3 & 6
\end{tabular}

TNM, tumor node metastasis.

sensitivity of $77.08 \%$ and $97.37 \%$ PPV. The sensitivity of $68 \mathrm{Ga}-$ DOTANOC PET-CT was not statistically different from that of FDG 18F-FDG PET-CT ( $p=0.109$ ). The combination of $68 \mathrm{Ga}-$ DOTANOC and 18F-FDG PET-CT could increase the sensitivity to $93.75 \%$.

For 6 cases with G3 primary tumors, 68Ga-DOTANOC PETCT identified 4 of these 6 patients and showed false negatives in 2 cases, while 18F-FDG PET-CT diagnosed 5 of these 6 patients and reported false negatives in one case (Figure 2). At the same time, among 42 patients with G1-2 primary tumors, 68Ga-DOTANOC PET-CT identified 39 patients (39/42), while 18 F-FDG PET-CT identified only 32 patients $(32 / 42)$ ( $\mathrm{p}=0.039)$.

\section{The Value of 68Ga-DOTANOC and 18F-FDG PET-CT in Predicting LN Group Metastasis}

Among the 11 patients who underwent regional lymphadenectomy, 10 patients were pathologically diagnosed with LN group metastasis. Forty-two groups of regional LNs were harvested after surgery, of which 18 groups were diagnosed with metastases. More details are shown in Table 2.

\section{Visual Assessment}

68Ga-DOTANOC PET-CT was true positive in $14 \mathrm{LN}$ groups and true negative in $22 \mathrm{LN}$ groups; thus, the sensitivity of $68 \mathrm{Ga}$ DOTANOC PET-CT for detecting RNETs was $77.78 \%$, and the specificity was $91.67 \%$. Meanwhile, 18F-FDG PET-CT was true positive in $7 \mathrm{LN}$ groups and true negative in $24 \mathrm{LN}$ groups, with $38.89 \%$ sensitivity and $100 \%$ specificity. The overall sensitivity, specificity, and accuracy of $68 \mathrm{Ga}-\mathrm{DOTANOC}$ and $18 \mathrm{~F}-\mathrm{FDG}$ PET-CT in predicting LN group metastasis are presented in Table 3.

Among the 18 positive LN groups, both 68Ga-DOTANOC PET-CT and 18F-FDG PET-CT were true positive in $7 \mathrm{LN}$ groups. Among the 24 negative LN groups, 18F-FDG PETCT defined all true negatives $(24 / 24)$, while $68 \mathrm{Ga}$ DOTANOCPET-CT assessed 2 of $24 \mathrm{LN}$ groups as false positives $(2 / 24)$. Both $68 \mathrm{Ga}-\mathrm{DOTANOC}$ and 18F-FDG PETCT were positive in $7 \mathrm{LN}$ groups and negative in $22 \mathrm{LN}$ groups. However, discordance was noted in 13 groups between the two tracers (Figure 3).
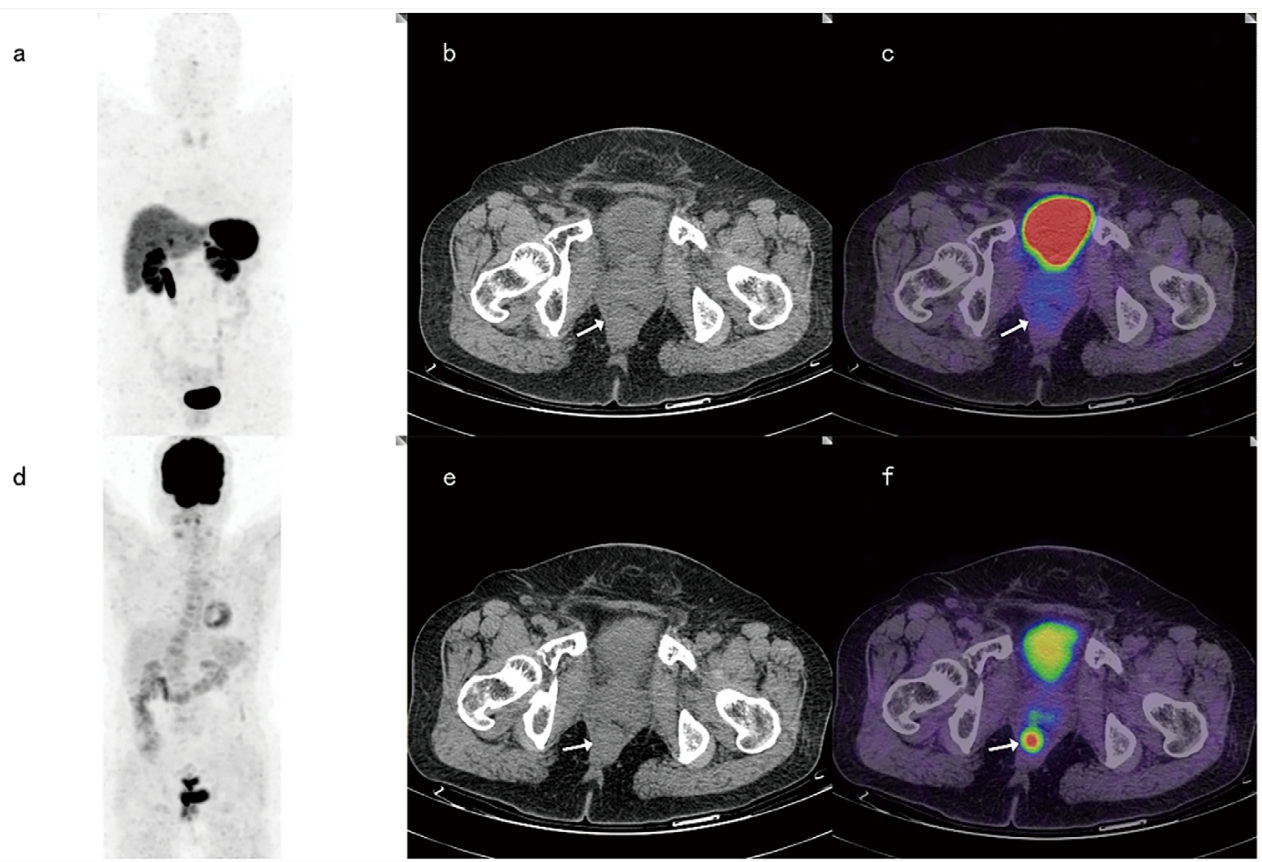

FIGURE 2 | A 65-year-old female patient with rectal neuroendocrine carcinoma (Ki-67 was 90\%). 68Ga-DOTANOC PET-CT images and corresponding MIP images (A-C) showed no focal uptake of 68Ga-DOTANOC, while 18F-FDG PET-CT imaging (D-F) showed focal uptake of 18F-FDG. 
TABLE 2 | General characteristics of patients who underwent region LN dissection.

\begin{tabular}{lc}
\hline Variables & Value \\
\hline Sex & \\
Male & 6 \\
Female & 5 \\
Age (years) & $38-70$ \\
TNM stage & \\
I-III & 6 \\
IV & 5 \\
Grade & \\
G1 & 2 \\
G2 & 8 \\
G3 & 1 \\
LN group metastases & \\
Positive & 18 \\
Negative & 24
\end{tabular}

TNM, tumor node metastasis; LN, Lymph node.

\section{Semiquantitative Assessment}

ROC analysis showed that the optimal SUVmax cut-off value with the highest accuracy for predicting malignant nodes through 68Ga-DOTANOC PET was 2.25 with $77.78 \%$ sensitivity, 91.67\% specificity, 87.50\% PPV, 84.62\% NPV and 85.71\% accuracy. The AUC was 0.824 (95\%CI:0.723-0.981)

(Figure 4A and Table 4).

Meanwhile, the optimal SUVmax cut-off value with the highest accuracy for predicting malignant nodes through $18 \mathrm{~F}$ FDG PET was 1.05 with $61.11 \%$ sensitivity, $75.00 \%$ specificity, 64.71\% PPV,72.00\% NPV and 69.05\% accuracy. The AUC was 0.664 (95\%CI: 0.485-0.844) (Figure 4B and Table 4).

\section{DISCUSSION}

This was the first study to evaluate the impact of $68 \mathrm{Ga}$ DOTANOC and 18F-FDG PET-CT in predicting LN metastasis in patients with RNETs. Our study showed that $68 \mathrm{Ga}$-DOTANOC PET-CT showed prospective ability to predict LN metastasis through visual assessment and semiquantitative assessment, which was better than 18F-FDG PET-CT. Meanwhile, the sensitivity of $68 \mathrm{Ga}-\mathrm{DOTANOC}$ in primary tumors were better than those of 18F-FDG PET-CT.

In our study, the overall sensitivity of $68 \mathrm{Ga}-\mathrm{DOTANOC}$ PET-CT was $89.58 \%$, and the PPV was $95.56 \%$ in primary tumors. Even for G3 tumors, 4/6 of primary tumors could be detected by $68 \mathrm{Ga}-\mathrm{DOTANOC}$ PET-CT. Several previous studies have shown that the sensitivity of 68GA-DOTANOC PET-CT for detecting gastrointestinal pancreatic primary lesions was $71.4 \%-94.4 \%$ (30-32), suggesting that 68Ga-DOTANOC had good diagnostic sensitivity. Meanwhile, 18F-FDG PET-CT identified $37 / 48$ primary tumors with $77.08 \%$ sensitivity in our research, which was similar to the results of Zhang, P et al. (33). Other studies have shown that 18F-FDG PET-CT was not sensitive for diagnosing NETs (sensitivity 33\%-66.7\%) (19, 34, 35 ). In our study, the sensitivity of $68 \mathrm{Ga}-\mathrm{DOTANOC}$ PET-CT was higher than that of 18F-FDG PET-CT for patients with G1G2 tumors $(\mathrm{p}=0.039)$, indicating that 68Ga-DOTANOC was more reliable for the diagnosis of G1-G2 RNETs.

We also found that the combination of 68Ga-DOTANOC and 18F-FDG PET-CT slightly increased the sensitivity to $93.75 \%$ in detecting primary tumors. Similarly, a study by Partelli, S. et al. (22) also showed that 68Ga-DOTANOC PET-CT combined with 18F-FDG PET-CT could only slightly increase the sensitivity in pancreatic neuroendocrine tumors, suggesting that $18 \mathrm{~F}-\mathrm{FDG}$ PET-CT is unnecessary for detecting RNETs.

The current literature evaluating 68Ga-DOTANOC PET-CT for diagnosing LN metastasis is limited. Our research showed that 68Ga-DOTANOC PET-CT had 77.78\% sensitivity and $91.67 \%$ specificity in both visual assessment and semiquantitative assessment for diagnosing LN group metastasis in RNETs, showing that the two evaluation methods were highly consistent. As reported by Ansquer, C (36). 68Ga-DOTANOC PET-CT had a sensitivity of $86.4 \%$ in midgut neuroendocrine tumors, and Niraj Naswa reported that $68 \mathrm{Ga}-\mathrm{DOTANOC}$ had a sensitivity of $92.8 \%$ and specificity of $100 \%$ in diagnosing LN metastasis for gastroenteropancreatic neuroendocrine tumors (23), indicating that $68 \mathrm{Ga}-\mathrm{DOTANOC}$ was a good tool for screening LN metastasis.

A study by Majala S. showed that only $33 \%$ of LN metastases could be diagnosed through 18F-FDG PET-CT in nonfunctional pancreatic neuroendocrine tumors (24). Meanwhile, another study showed that 68Ga-DOTANOC PET-CT had $94.2 \%$ sensitivity, $87.5 \%$ specificity, and $92.1 \%$ accuracy while $18 \mathrm{~F}$ FDG PET-CT had $25.7 \%$ sensitivity, $100 \%$ specificity, and $49 \%$ accuracy in gastroenteropancreatic neuroendocrine tumors (23). In concordance with the above research, our research showed that the sensitivity of 18F-FDG PET-CT for detecting LN group metastasis was only $38.89 \%$ in visual assessment and $61.11 \%$ in semiquantitative assessment with an AUC of 0.664 , which was lower than the sensitivity and AUC of $68 \mathrm{Ga}$ DOTANOC PET-CT (sensitivity was $77.78 \%$ and the AUC was 0.854$)$. Therefore, we concluded that $68 \mathrm{Ga}$-DOTANOC

TABLE 3 | Sensitivity, specificity, positive and negative predictive value and accuracy for the prediction of LN group metastasis by 68Ga-DOTANOC and 18F-FDG PET through visual assessment.

\begin{tabular}{lccc}
\hline & 68Ga-DOTANOC PET & 18F-FDG PET & p value \\
\hline Sensitivity (\%) (95\% Cl) & $77.78(52.36-93.59)$ & $38.89(17.30-64.25)$ & 100 \\
Specificity (\%) (95\% Cl) & $91.67(73.00-98.97)$ & 100 & 0.018 \\
PPV (\%) (95\% Cl) & $87.5(64.48-96.43)$ & $69.57(55.20-80.92)$ & 1.000 \\
NPV (\%) (95\% Cl) & $84.62(69.68-92.94)$ & $68.57(60.15-75.93)$ & 0.150 \\
Accuracy (\%) (95\% Cl) & $85.71(71.46-94.57)$ & & 0.175 \\
\hline
\end{tabular}

PPV, positive predictive value; NPV, negative predictive value. 


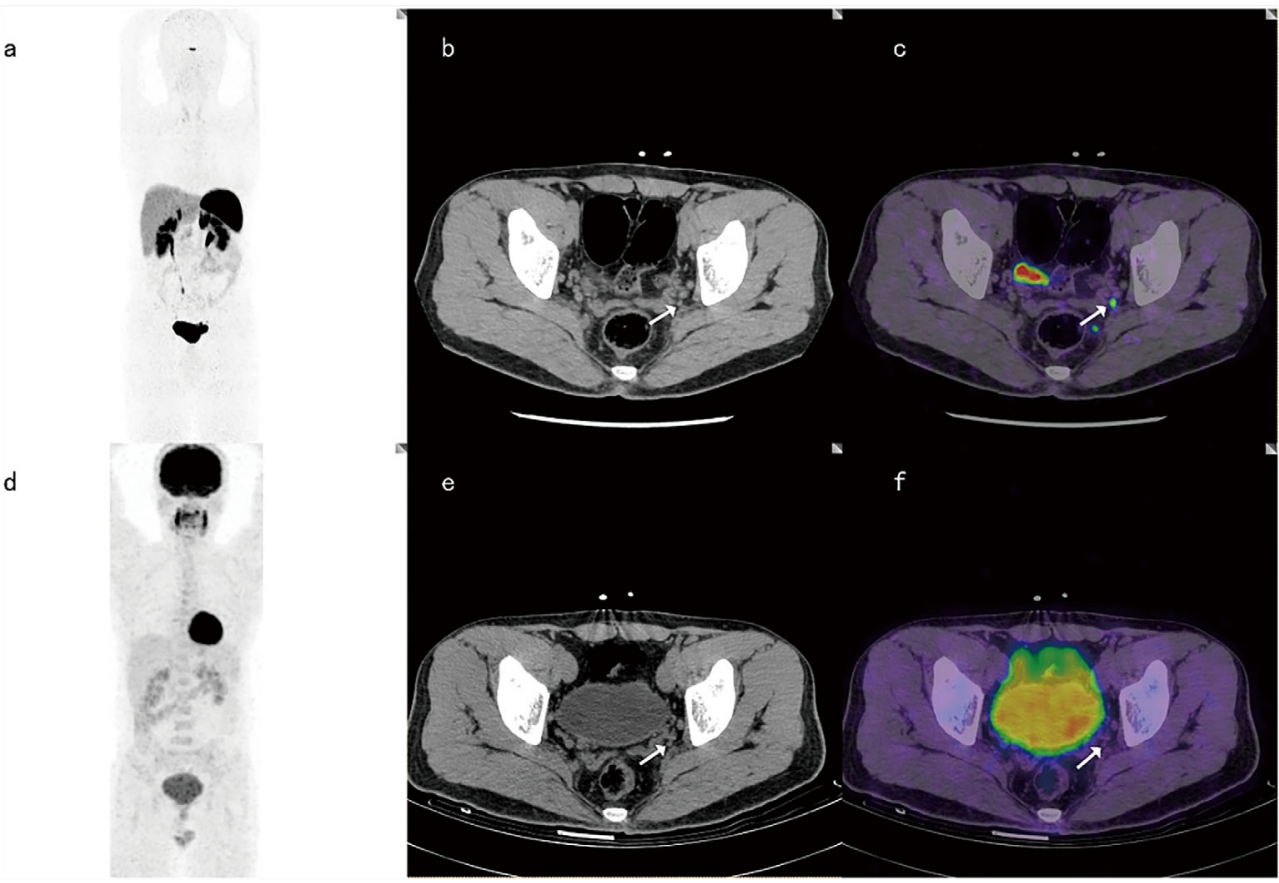

FIGURE 3 | A 37-year-old male with LN metastasis adjacent to the left iliac blood vessel. The focal uptake of 68Ga-DOTANOC in LNs (as shown by the arrow) was obviously increased (A-C), while the focal uptake of 18F-FDG was similar to that in the background (D-F).
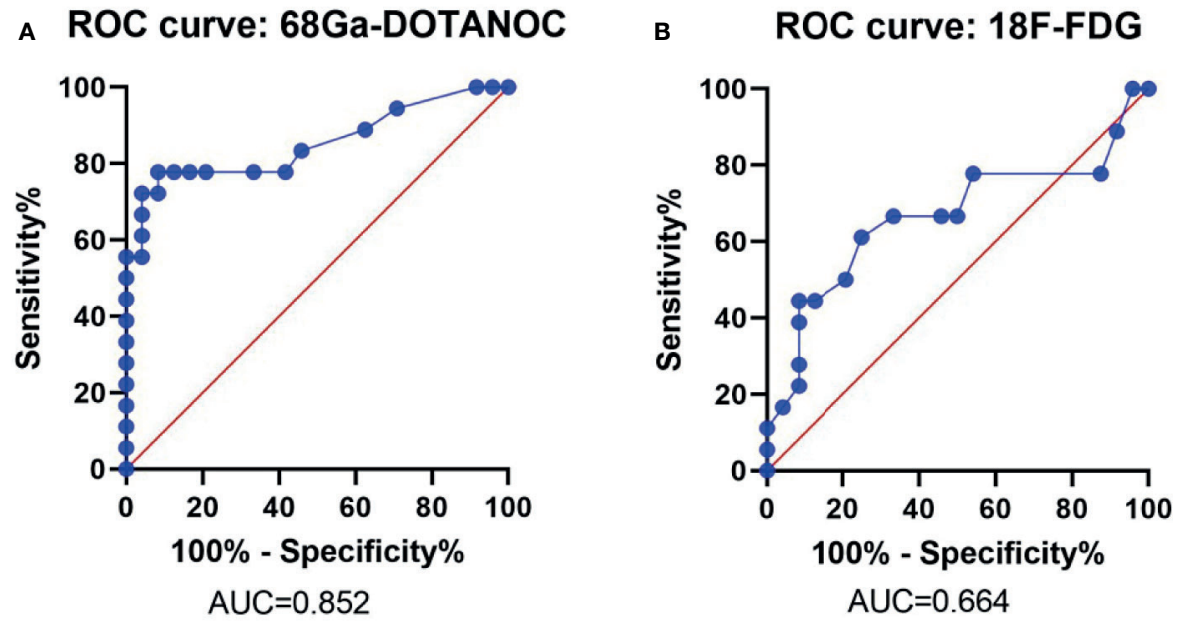

FIGURE 4 | ROC Curve of the SUVmax of 68Ga-DOTANOC and 18F-FDG PET in predicting LN group metastasis. ROC Curve of 68Ga-DOTANOC PET, The AUC was 0.852 (A); ROC Curve of 18F-FDG PET. The AUC was 0.664 (B). ROC, Receiver Operating Characteristics; AUC, The area under the ROC curves.

PET-CT was more suitable for screening LN group metastasis than 18 F-FDG PET-CT.

68Ga-DOTANOC and 18F-FDG PET-CT had similar diagnostic capabilities in evaluating primary tumors but had different diagnostic capabilities in LN metastases. The result may be due to the active lymph node inflammation in the rectal network (37), which affects FDG uptake (38) and cause FDG PET is not reliable for detecting LN metastases of RNETs or maybe $68 \mathrm{Ga}-\mathrm{DOTANOC}$ is actually better than FDG as N. Naswa reported (23). 
TABLE 4 | Sensitivity, specificity, positive and negative predictive value, accuracy and AUC for the prediction of LN group metastasis by 68Ga-DOTANOC and 18F-FDG PET through semiquantitative assessment.

\begin{tabular}{lccc}
\hline & 68Ga-DOTANOC PET & 18F-FDG PET & p value \\
\hline Sensitivity (\%) (95\% Cl) & $77.78(52.36-93.59)$ & $61.11(35.75-82.70)$ & 0.278 \\
Specificity (\%) (95\% Cl) & $91.67(73.00-98.97)$ & $75.00(53.29-90.23)$ & 0.121 \\
PPV (\%) (95\% Cl) & $87.50(64.48-96.43)$ & $64.71(45.54-80.08)$ & 0.127 \\
NPV (\%) (95\% Cl) & $84.62(69.68-92.94)$ & $72.00(57.96-82.75)$ & 0.274 \\
Accuracy (\%) (95\% Cl) & $85.71(71.46-94.57)$ & $69.05(52.91-82.38)$ & 0.015 \\
AUC (95\% Cl) & $0.852(0.723-0.981)$ & $0.664(0.485-0.844)$ & 0.0583
\end{tabular}

PPV, positive predictive value; NPV, negative predictive value; AUC, Area under ROC curve.

Usually, in parallel with an increasing tumor proliferation rate (Ki-67 index), 68 Ga-DOTA- somatostatin receptor expression in NETs decreases (39). For NETs with Ki-67 greater than $15 \%$, metabolic imaging with 18 FDG PET-CT is usually preferred rather than $68 \mathrm{Ga}$-DOTA-somatostatin analog PET-CT because of the low or absent somatostatin receptor expression in NET lesions (40). In our study, most patients had Ki-67 less than $15 \%$, which may explain why the performance of 68Ga-DOTANOC PET-CT was better than 18F-FDG PET-CT. Therefore, 68Ga-DOTANOC PET-CT seems to be more suitable for RNET assessment than 18F-FDG PET-CT, especially in G1G2 RNETs.

With the emergence of molecular imaging, surgeons are using molecular imaging to image-guided surgery (41). Hybrid detection modalities for image-guided surgery has been applied such as the application of indocyanine green (ICG)-99mTcnanolloid for cancers (42). Similarly, Håkan Orlefors reported that 11C-5-Hydroxytryptophane PET can localize the NETs (43). However, the hybrid detection modalities for imageguided surgery in NETs are rare. SSTRs such as 68GaDOTANOC have high specificity, but whether it is suitable for guided surgery needs further study; meanwhile, combination of image-guided surgery and robot-assisted with laparoscopic surgery may reduce surgical complications in the future (44).

The strengths of the present study are that we evaluated simultaneously the primary tumors and regional LN group

\section{REFERENCES}

1. Moertel CG. Karnofsky Memorial Lecture. An Odyssey in the Land of Small Tumors. J Clin Oncol (1987) 5:1502-22. doi: 10.1200/JCO.1987.5.10.1502

2. Ahmed M. Gastrointestinal Neuroendocrine Tumors in 2020. World J Gastrointest Oncol (2020) 12:791-807. doi: 10.4251/wjgo.v12.i8.791

3. Maggard MA, O'Connell JB, Ko CY. Updated Population-Based Review of Carcinoid Tumors. Ann Surg (2004) 240:117-22. doi: 10.1097/01. sla.0000129342.67174.67

4. Kim JY, Hong S-M, Ro JY. Recent Updates on Grading and Classification of Neuroendocrine Tumors. Ann Diagn Pathol (2017) 29:11-6. doi: 10.1016/ j.anndiagpath.2017.04.005

5. Hauso O, Gustafsson BI, Kidd M, Waldum HL, Drozdov I, Chan AKC, et al. Neuroendocrine Tumor Epidemiology: Contrasting Norway and North America. Cancer (2008) 113:2655-64. doi: 10.1002/cncr.23883

6. Garcia-Carbonero R, Capdevila J, Crespo-Herrero G, Díaz-Pérez JA, Martínez Del Prado MP, Alonso Orduña V, et al. Incidence, Patterns of Care and Prognostic Factors for Outcome of Gastroenteropancreatic histologically of RNETs. However, there are some limitations. Firstly, the major limitation of the present study was the number of patients, as RNETs are rare; therefore, the conclusion should be treated carefully, and more cases need to be studied in the future. Secondly, as the study was retrospective, RCT research is needed.

\section{DATA AVAILABILITY STATEMENT}

The original contributions presented in the study are included in the article/supplementary material. Further inquiries can be directed to the corresponding author.

\section{ETHICS STATEMENT}

The studies involving human participants were reviewed and approved by Ethics Committee of the First Affiliated Hospital of Sun Yat-sen University. The patients/participants provided their written informed consent to participate in this study.

\section{AUTHOR CONTRIBUTIONS}

ZW and GL designed this study. ZZ interpreted the patient data and drafted the manuscript. BZ and YW contributed to data collection. GL and ZXW revised the manuscript. All authors contributed to the article and approved the submitted version.

\section{FUNDING}

This study was funded by the Natural Science Foundation of Guangdong Province, China (Grant Nos. 2016A030310155, 2017A030313577, and 2018A030313978) and the National Natural Science Foundation of China (Grant Nos. 81602049 and 81802342).
Neuroendocrine Tumors (GEP-NETs): Results From the National Cancer Registry of Spain (RGETNE). Ann Oncol (2010) 21:1794-803. doi: 10.1093/ annonc/mdq022

7. Yao JC, Hassan M, Phan A, Dagohoy C, Leary C, Mares JE, et al. One Hundred Years After "Carcinoid": Epidemiology of and Prognostic Factors for Neuroendocrine Tumors in 35,825 Cases in the United States. J Clin Oncol (2008) 26:3063-72. doi: 10.1200/JCO.2007.15.4377

8. Masui T, Ito T, Komoto I, Uemoto S. Recent Epidemiology of Patients With Gastro-Entero-Pancreatic Neuroendocrine Neoplasms (GEP-NEN) in Japan: A Population-Based Study. BMC Cancer (2020) 20:1104. doi: 10.1186/s12885020-07581-y

9. Bertani E, Ravizza D, Milione M, Massironi S, Grana CM, Zerini D, et al. Neuroendocrine Neoplasms of Rectum: A Management Update. Cancer Treat Rev (2018) 66:45-55. doi: 10.1016/j.ctrv.2018.04.003

10. Goretzki PE, Mogl MT, Akca A, Pratschke J. Curative and Palliative Surgery in Patients With Neuroendocrine Tumors of the Gastro-Entero-Pancreatic (GEP) Tract. Rev Endocr Metab Disord (2018) 19:169-78. doi: 10.1007/ s11154-018-9469-9 
11. Andreasi V, Partelli S, Muffatti F, Manzoni MF, Capurso G, Falconi M. Update on Gastroenteropancreatic Neuroendocrine Tumors. Dig Liver Dis (2021) 53:171-82. doi: 10.1016/j.dld.2020.08.031

12. de Mestier L, Lorenzo D, Fine C, Cros J, Hentic O, Walter T, et al. Endoscopic, Transanal, Laparoscopic, and Transabdominal Management of Rectal Neuroendocrine Tumors. Best Pract Res Clin Endocrinol Metab (2019) 33:101293. doi: 10.1016/j.beem.2019.101293

13. Refardt J, Hofland J, Kwadwo A, Nicolas GP, Rottenburger C, Fani M, et al. Theranostics in Neuroendocrine Tumors: An Overview of Current Approaches and Future Challenges. Rev Endocr Metab Disord (2020) 22:581-4. doi: 10.1007/s11154-020-09552-x

14. Sundin A, Arnold R, Baudin E, Cwikla JB, Eriksson B, Fanti S, et al. ENETS Consensus Guidelines for the Standards of Care in Neuroendocrine Tumors: Radiological, Nuclear Medicine \& Hybrid Imaging. Neuroendocrinology (2017) 105:212-44. doi: 10.1159/000471879

15. Sanli Y, Garg I, Kandathil A, Kendi T, Zanetti MJB, Kuyumcu S, et al. Neuroendocrine Tumor Diagnosis and Management: Ga-DOTATATE PET/Ct. AJR Am J Roentgenol (2018) 211:267-77. doi: 10.2214/AJR. 18.19881

16. Tirosh A, Kebebew E. The Utility of Ga-DOTATATE Positron-Emission Tomography/Computed Tomography in the Diagnosis, Management, Follow-Up and Prognosis of Neuroendocrine Tumors. Future Oncol (2018) 14:111-22. doi: 10.2217/fon-2017-0393

17. Carideo L, Prosperi D, Panzuto F, Magi L, Pratesi MS, Rinzivillo M, et al. Role of Combined [Ga]Ga-DOTA-SST Analogues and [F]FDG PET/CT in the Management of GEP-NENs: A Systematic Review. J Clin Med (2019) 8:1032. doi: $10.3390 / j \mathrm{~cm} 8071032$

18. Deroose CM, Hindié E, Kebebew E, Goichot B, Pacak K, Taïeb D, et al. Molecular Imaging of Gastroenteropancreatic Neuroendocrine Tumors: Current Status and Future Directions. J Nucl Med (2016) 57:1949-56. doi: 10.2967/jnumed.116.179234

19. Rinzivillo M, Partelli S, Prosperi D, Capurso G, Pizzichini P, Iannicelli E, et al. Clinical Usefulness of (18) F-Fluorodeoxyglucose Positron Emission Tomography in the Diagnostic Algorithm of Advanced Entero-Pancreatic Neuroendocrine Neoplasms. Oncologist (2018) 23:186-92. doi: 10.1634/ theoncologist.2017-0278

20. Alevroudis E, Spei M-E, Chatziioannou SN, Tsoli M, Wallin G, Kaltsas G, et al. Clinical Utility of F-FDG PET in Neuroendocrine Tumors Prior to Peptide Receptor Radionuclide Therapy: A Systematic Review and MetaAnalysis. Cancers (Basel) (2021) 13:1813. doi: 10.3390/cancers13081813

21. Imperiale A, Meuter L, Pacak K, Taïeb D. Variants and Pitfalls of PET/CT in Neuroendocrine Tumors. Semin Nucl Med (2021) 51:519-28. doi: 10.1053/ j.semnuclmed.2021.03.001

22. Partelli S, Rinzivillo M, Maurizi A, Panzuto F, Salgarello M, Polenta V, et al. The Role of Combined Ga-DOTANOC and (18)FDG PET/CT in the Management of Patients With Pancreatic Neuroendocrine Tumors. Neuroendocrinology (2014) 100:293-9. doi: 10.1159/000368609

23. Naswa N, Sharma P, Gupta SK, Karunanithi S, Reddy RM, Patnecha M, et al. Dual Tracer Functional Imaging of Gastroenteropancreatic Neuroendocrine Tumors Using 68Ga-DOTA-NOC PET-CT and 18F-FDG PET-CT: Competitive or Complimentary? Clin Nucl Med (2014) 39:e27-34. doi: 10.1097/RLU.0b013e31827a216b

24. Majala S, Seppänen H, Kemppainen J, Sundström J, Schalin-Jäntti C, Gullichsen R, et al. Prediction of the Aggressiveness of Non-Functional Pancreatic Neuroendocrine Tumors Based on the Dual-Tracer PET/Ct. EJNMMI Res (2019) 9:116. doi: 10.1186/s13550-019-0585-7

25. Ayati N, Lee ST, Zakavi R, Pathmaraj K, Al-Qatawna L, Poon A, et al. Long-Acting Somatostatin Analog Therapy Differentially Alters GaDOTATATE Uptake in Normal Tissues Compared With Primary Tumors and Metastatic Lesions. J Nucl Med (2018) 59:223-7. doi: 10.2967/jnumed.117.192203

26. Nagtegaal ID, Odze RD, Klimstra D, Paradis V, Rugge M, Schirmacher P, et al. The 2019 WHO Classification of Tumours of the Digestive System. Histopathology (2020) 76:182-8. doi: 10.1111/his.13975

27. Yang M, Zeng L, Yao WQ, Ke NW, Tan CL, Tian BL, et al. A Comprehensive Validation of the Novel 8th Edition of American Joint Committee on Cancer Staging Manual for the Long-Term Survivals of Patients With non-Functional
Pancreatic Neuroendocrine Neoplasms. Med (Baltimore) (2020) 99:e22291. doi: $10.1097 / \mathrm{md} .0000000000022291$

28. Japanese Society for Cancer of the Colon and Rectum. Japanese Classification of Colorectal, Appendiceal, and Anal Carcinoma: The 3D English Edition [Secondary Publication]. J Anus Rectum Colon (2019) 3:175-95. doi: 10.23922/jarc.2019-018

29. He Q, Zhang B, Zhang L, Chen Z, Shi X, Yi C, et al. Diagnostic Efficiency of Ga-DOTANOC PET/CT in Patients With Suspected Tumour-Induced Osteomalacia. Eur Radiol (2021) 31:2414-21. doi: 10.1007/s00330-02007342-2

30. Wild D, Bomanji JB, Benkert P, Maecke H, Ell PJ, Reubi JC, et al. Comparison of $68 \mathrm{Ga}$-DOTANOC and $68 \mathrm{Ga}$-DOTATATE PET/CT Within Patients With Gastroenteropancreatic Neuroendocrine Tumors. J Nucl Med (2013) 54:36472. doi: 10.2967/jnumed.112.111724

31. Sharma A, Das CJ, Makharia GK, Arora G, Kumar R. Comparison of Contrast-Enhanced CT + CT Enterography and 68Ga-DOTANOC PET/CT in Gastroenteropancreatic Neuroendocrine Tumors. Clin Nucl Med (2020) 45:848-53. doi: 10.1097/RLU.0000000000003188

32. Naswa N, Sharma P, Kumar A, Nazar AH, Kumar R, Chumber S, et al. Gallium-68-DOTA-NOC PET/CT of Patients With Gastroenteropancreatic Neuroendocrine Tumors: A Prospective Single-Center Study. AJR Am J Roentgenol (2011) 197:1221-8. doi: 10.2214/AJR.11.7298

33. Zhang P, Yu J, Li J, Shen L, Li N, Zhu H, et al. Clinical and Prognostic Value of PET/CT Imaging With Combination of (68)Ga-DOTATATE and (18)F-FDG in Gastroenteropancreatic Neuroendocrine Neoplasms. Contrast Media Mol Imaging (2018) 2018:2340389. doi: 10.1155/2018/2340389

34. Has Simsek D, Kuyumcu S, Turkmen C, Sanl Y, Aykan F, Unal S, et al. Can Complementary 68Ga-DOTATATE and 18F-FDG PET/CT Establish the Missing Link Between Histopathology and Therapeutic Approach in Gastroenteropancreatic Neuroendocrine Tumors? J Nucl Med (2014) 55:1811-7. doi: 10.2967/jnumed.114.142224

35. Kayani I, Bomanji JB, Groves A, Conway G, Gacinovic S, Win T, et al. Functional Imaging of Neuroendocrine Tumors With Combined PET/CT Using 68Ga-DOTATATE (DOTA-DPhe1,Tyr3-Octreotate) and 18F-FDG. Cancer (2008) 112:2447-55. doi: 10.1002/cncr.23469

36. Ansquer C, Touchefeu Y, Faivre-Chauvet A, Leux C, Le Bras M, Régenet N, et al. Head-To-Head Comparison of 18F-DOPA PET/CT and $68 \mathrm{Ga}$ DOTANOC PET/CT in Patients With Midgut Neuroendocrine Tumors. Clin Nucl Med (2021) 46:181-6. doi: 10.1097/RLU.0000000000003450

37. Pijl JP, Nienhuis PH, Kwee TC, Glaudemans AWJM, Slart RHJA, Gormsen LC. Limitations and Pitfalls of FDG-PET/CT in Infection and Inflammation. Semin Nucl Med (2021) S0001-2998(21)00040-4. doi: 10.1053/j.semnuclmed. 2021.06.008

38. Kubota K. From Tumor Biology to Clinical Pet: A Review of Positron Emission Tomography (PET) in Oncology. Ann Nucl Med (2001) 15:47186. doi: 10.1007/BF02988499

39. Sundin A. Novel Functional Imaging of Neuroendocrine Tumors. Endocrinol Metab Clin North Am (2018) 47:505-23. doi: 10.1016/j.ecl.2018.04.003

40. Binderup T, Knigge U, Loft A, Mortensen J, Pfeifer A, Federspiel B, et al. Functional Imaging of Neuroendocrine Tumors: A Head-to-Head Comparison of Somatostatin Receptor Scintigraphy, 123I-MIBG Scintigraphy, and 18F-FDG PET. J Nucl Med (2010) 51:704-12. doi: 10.2967/jnumed.109.069765

41. Mondal SB, O’Brien CM, Bishop K, Fields RC, Margenthaler JA, Achilefu S. Repurposing Molecular Imaging and Sensing for Cancer Image-Guided Surgery. J Nucl Med (2020) 61:1113-22. doi: 10.2967/jnumed.118.220426

42. Van Oosterom MN, Rietbergen DDD, Welling MM, van der Poel HG, Maurer T, Van Leeuwen FWB. Recent Advances in Nuclear and Hybrid Detection Modalities for Image-Guided Surgery. Expert Rev Med Devices (2019) 16:71134. doi: 10.1080/17434440.2019.1642104

43. Orlefors H, Sundin A, Eriksson B, Skogseid B, Oberg K, Akerström G, et al. PET-Guided Surgery - High Correlation Between Positron Emission Tomography With 11C-5-Hydroxytryptophane (5-HTP) and Surgical Findings in Abdominal Neuroendocrine Tumours. Cancers (Basel) (2012) 4:100-12. doi: 10.3390/cancers4010100

44. Meershoek P, van Oosterom MN, Simon H, Mengus L, Maurer T, van Leeuwen PJ, et al. Robot-Assisted Laparoscopic Surgery Using DROP-IN 
Radioguidance: First-in-Human Translation. Eur J Nucl Med Mol Imaging (2019) 46:49-53. doi: 10.1007/s00259-018-4095-Z

Conflict of Interest: The authors declare that the research was conducted in the absence of any commercial or financial relationships that could be construed as a potential conflict of interest.

Publisher's Note: All claims expressed in this article are solely those of the authors and do not necessarily represent those of their affiliated organizations, or those of the publisher, the editors and the reviewers. Any product that may be evaluated in this article, or claim that may be made by its manufacturer, is not guaranteed or endorsed by the publisher.

Copyright (c) 2021 Zhou, Wang, Zhang, Wu, Li and Wang. This is an open-access article distributed under the terms of the Creative Commons Attribution License (CC BY). The use, distribution or reproduction in other forums is permitted, provided the original author(s) and the copyright owner(s) are credited and that the original publication in this journal is cited, in accordance with accepted academic practice. No use, distribution or reproduction is permitted which does not comply with these terms. 